PSICOLOGÍA

IBEROAMERICANA
Psicología Iberoamericana ISSN: 1405-0943

revista.psicologia@ibero.mx

Universidad Iberoamericana, Ciudad de México México

\title{
Fortalezas en adolescentes que no consumen tabaco
}

Sánchez-Xicotencatl, Carlos Omar; Andrade Palos, Patricia

Fortalezas en adolescentes que no consumen tabaco

Psicología Iberoamericana, vol. 27, núm. 2, 2019

Universidad Iberoamericana, Ciudad de México, México

Disponible en: http://www.redalyc.org/articulo.oa?id=133962309003 


\title{
Fortalezas en adolescentes que no consumen tabaco
}

\author{
Assets in adolescents who do not use tobacco \\ Carlos Omar Sánchez-Xicotencatl \\ omarxicotencatl@hotmail.com \\ Universidad Nacional Autónoma de México (UNAM), México \\ (D) http://orcid.org/0000-0002-7767-0040 \\ Patricia Andrade Palos \\ Universidad Nacional Autónoma de México (UNAM), México \\ http://orcid.org/0000-003-0072-1169
}

Psicología Iberoamericana, vol. 27, núm. 2, 2019

Universidad Iberoamericana, Ciudad de México, México

Recepción: 18 Enero 2019 Aprobación: 21 Octubre 2019

Redalyc: http://www.redalyc.org/ articulo.oa?id=133962309003

Financiamiento

Fuente: El proyecto CONACYT 247574

"Programa de promoción de la salud en estudiantes de secundaria”.

$\mathrm{N}^{\circ}$ de contrato: 247574
Resumen: En el presente estudio se analizaron las fortalezas internas y externas que protegen a los adolescentes del consumo de tabaco. El diseño de la investigación fue no experimental ex post facto, participaron 1567 estudiantes de secundaria y bachillerato de la Ciudad de México. Se utilizó la escala de Fortalezas Internas y Externas para Adolescentes (FIE-A). Se realizaron análisis descriptivos y de regresión logística, lo que permitió observar que, en los estudiantes de secundaria y preparatoria, la resistencia a la presión de pares disminuye la probabilidad del consumo. Para los estudiantes de secundaria las fortalezas protectoras fueron responsabilidad, supervisión de la madre, amigos sin conductas de riesgo y no acceso a drogas, y en los estudiantes de preparatoria fueron importancia de la salud y evitación de conductas de riesgo. Estos resultados confirman la importancia de hacer análisis por grupos de edad y sirven para diseñar intervenciones preventivas basadas en evidencia.

Palabras clave: Fortalezas internas, fortalezas externas, estudiantes, consumo de tabaco. Abstract: In this study, internal and external assets that protect adolescents from tobacco use were analyzed. The research design was non-experimental ex post facto and 1567 junior high school and high school students from Mexico City participated. The scale of Internal and External Strengths for Adolescents (FIE-A) was used. Descriptive and logistic regression analysis was used. The analysis allowed us to observe that, in junior and high school students, resistance to peer pressure decreases the probability of consumption. Protective factors such as responsibility, supervision of the mother, friends without risk behaviors and no access to drugs, were found amongst junior and senior high school students. Factors such as importance of health and avoidance of risk behaviors were also important. These results confirm the relevance of sampling different age groups and will help create evidence-based interventions to prevent tobacco use.

Keywords: Internal assets, tobacco consumption.

\section{Introducción}

El consumo de tabaco es un problema de salud que afecta a muchos países del mundo; por ejemplo, en Estados Unidos de América se estima que $16 \%$ de la mortalidad es atribuible al consumo de este producto, y muchas de estas defunciones se relacionan con cáncer, enfermedades cardiovasculares, enfermedades crónicas respiratorias y con la exposición al humo de tabaco (Organización Panamericana de la Salud, 2018). Si bien en México se ha observado una disminución en el consumo de tabaco en los últimos años ( $\mathrm{Ng}$ et al., 2014), el tabaquismo sigue 
siendo la principal causa de muerte prevenible, y los costos que genera en el sistema de salud son elevados. Específicamente en adolescentes, la Encuesta Nacional de Consumo de Drogas en Estudiantes reporta que $30.4 \%$ de estudiantes de secundaria y bachillerato han consumido tabaco alguna vez en la vida, siendo los hombres los que presentan un mayor porcentaje (34\%) en comparación con las mujeres (26.8\%); en promedio los jóvenes inician el consumo entre los 13 y 14 años. Cuando se analizan los resultados por grado escolar, se observa que conforme aumenta la edad, el consumo de tabaco incrementa considerablemente. Por ejemplo, el reporte de consumo alguna vez en la vida de hombres de secundaria fue de $23.8 \%$ y en bachillerato de $51.4 \%$; por su parte, se observa un incremento en mujeres que va de un $17.7 \%$ en secundaria a $41.3 \%$ en bachillerato. A su vez, el consumo en el último mes en hombres pasó de $7.4 \%$ a $23.1 \%$ y en las mujeres de $5.2 \%$ a $14.8 \%$, mientras que el consumo diario también se incrementó significativamente, ya que los hombres de secundaria reportaron un $5.7 \%$ comparado con un $14.7 \%$ en bachillerato, consumo que en las mujeres cambió de 3.9\% a 8.7\% (Instituto Nacional de Psiquiatría, Comisión Nacional contra las Adicciones, Secretaría de Salud, 2015).

Es extensa la literatura internacional que estudia los factores de riesgo para el consumo de tabaco en adolescentes; por ejemplo, algunos factores ambientales como la influencia de los amigos que consumen (Chun \& Chung, 2013; Jaber, Mzayek, Madhivanan, Khader, \& Maziak, 2016); padres consumidores (Kim \& Chun, 2016; Mays et al., 2014; Veeranki et al., 2015); así como algunos factores individuales como la búsqueda de sensaciones (Brikmanis, Petersen, \& Doran, 2017); las creencias asociadas al consumo de tabaco (Kleinjan, van den Eijnden, \& Engels, 2009), y la percepción de riesgo (Pilatti, Read, \& Pautassi, 2017). Si bien estas investigaciones tratan de explicar los factores que favorecen el consumo de tabaco, en los últimos años algunos trabajos se han enfocado en los aspectos que promueven conductas saludables en los jóvenes; por ejemplo, el enfoque de Desarrollo Positivo de los Jóvenes (PYD por sus siglas en inglés). Este enfoque analiza los factores que favorecen que los jóvenes tengan un desarrollo integral, es decir, que los adolescentes no sólo eviten las conductas de riesgo como el consumo de tabaco, sino que además incrementen otras habilidades para que logren un desarrollo integral.

En los últimos años se han desarrollado varios modelos de PYD (Heck \& Subramaniam, 2009). Uno de estos es el modelo de fortalezas propuesto por Benson (2007), el cual plantea que la interacción de factores individuales y contextuales promueve un desarrollo integral en los jóvenes. A estos factores los denomina fortalezas, las cuales se definen como relaciones clave, oportunidades, valores, habilidades y autopercepciones que ayudan a limitar el acercamiento del adolescente a conductas de riesgo, manifestar resiliencia ante las adversidades y de esta manera tener un desarrollo positivo. Estas fortalezas se clasifican en internas y externas (Benson, Scales, Hamilton, \& Sesma, 2006). Las internas hacen referencia a las características propias de los jóvenes (por ejemplo, responsabilidad, planeación y toma de decisiones, resistencia 
a la presión de otros); mientras que las externas se refieren a aspectos contextuales (por ejemplo, supervisión de los padres, amigos saludables, límites y expectativas).

Existen estudios que, si bien no se basan en la propuesta de Benson (2007), sí estudian factores que podrían ser considerados fortalezas internas y externas que protegen a los jóvenes del consumo de tabaco. Por ejemplo, cuando se estudia la influencia que tienen variables contextuales, Andrade y Sánchez-Xicotencatl (2018) en un estudio con adolescentes mexicanos encontraron que tener amigos que no presentan conductas de riesgo es un predictor importante del no consumo de tabaco tanto para hombres como para mujeres. Esto es consistente con la revisión de Kobus (2003) acerca de la influencia de los amigos en el consumo de tabaco, donde encontró que los jóvenes que tienen amigos que no fuman son menos propensos a consumir.

Otras fortalezas externas que se presentan en la familia también se asocian con el no consumo. Por ejemplo, la percepción de una buena relación de los adolescentes con los otros integrantes de su familia se relaciona con bajos niveles de consumo de tabaco. NuñoGutiérrez, Álvarez-Nemegyei, Velázquez-Castañeda y Tapia-Curiel (2008) encontraron que existe un ambiente familiar más favorable para los adolescentes no fumadores comparados con los fumadores, y DiNapoli (2009) menciona que platicar los problemas personales con los padres es un factor que disminuye el consumo de tabaco en los jóvenes. Además, cuando los jóvenes tienen padres que no fuman, se establecen reglas parentales claras en casa y supervisión de los hijos, lo cual disminuye el riesgo de consumo de tabaco en los hijos ( Piko \& Balázs, 2012 ). Asimismo, Kim y Chun (2016) en un estudio realizado en 31 países en vías de desarrollo encontraron que los jóvenes con una buena relación con sus padres y que eran supervisados, reportaban una baja frecuencia de consumo de tabaco. También Hiemstra, de Leeuw, Engels y Otten (2017) mencionan que los padres que prohíben el consumo de tabaco dentro de la casa disminuyen el consumo de los jóvenes.

El medio donde vive el adolescente también puede proteger a los jóvenes para que no consuman sustancias. Pabayo, O'Loughlin, Barnett, Cohen y Gauvin (2012) encontraron en jóvenes canadienses que la intolerancia para fumar dentro de la escuela o en lugares cercanos a la escuela disminuyen considerablemente el consumo de tabaco. En España, Lido\#n-Moyano et al. (2017) reportaron una disminución en el consumo de tabaco a partir de que se implementaron normas antitabaco.

Como se mencionó anteriormente, el grupo de amigos es muy importante para los adolescentes y, si bien en ocasiones se consumen sustancias debido a la presión que ejercen los pares, algunos jóvenes son capaces de resistir a esta presión, lo que los protege del consumo no sólo de tabaco sino de otras sustancias como el alcohol y la marihuana. Son diversos los estudios que mencionan que los adolescentes con altos niveles de resistencia a la presión de los amigos para consumir, presentan un menor consumo de sustancias (Andrade, Pérez, Alfaro, Sánchez, \& 
Montes, 2009; Gibbon, Griffin, Tanno, Tanigawa, \& Botvin, 2014; Hopfer, Hecht, Lanza, Xianming, \& Xu, 2013).

También se han identificado otros aspectos individuales que protegen al adolescente del consumo de tabaco, como la importancia que el joven le da a practicar una religión y asistir a servicios religiosos con frecuencia (Fletcher \& Kumar, 2014; Nonnemaker, McNeely, \& Blum, 2006; Sinha, Cnaan, \& Gelles, 2007), o la adecuada expresión del enojo, así como una buena toma de decisiones (Alfaro, Sánchez, Andrade, Pérez, \& Montes, 2010; Bernstein, Colby, Bidwell, Kahler, \& Leventhal, 2014).

Por otro lado, muchos adolescentes inician el consumo de tabaco debido a que creen que no es peligroso para la salud o que los daños provocados por el consumo sólo se presentan después de muchos años. Por lo anterior, algunos autores enfatizan la importancia de incrementar en los adolescentes la percepción del daño que ocasiona el tabaco, ya que conforme se incrementa dicha percepción el consumo disminuye (Cable, Roman, \& Kelly, 2017; Kasim, Al-Zalabani, Abd El-Moneim, \& Amer, 2016).

Como se puede apreciar, es amplia la literatura de factores que protegen al adolescente del consumo de tabaco; no obstante, pocos estudios se han realizado con el enfoque de fortalezas. Debido a esto, el objetivo de este estudio fue identificar las fortalezas internas y externas que protegen a los jóvenes del consumo de tabaco y la hipótesis central del trabajo fue que los jóvenes que no consumen tabaco presentan mayores puntajes en algunas fortalezas internas y externas.

\section{Método}

Se llevó a cabo un estudio no experimental ex post facto, en el cual las variables se evaluaron con base en la información proporcionada por los adolescentes. La pregunta de investigación plantea ¿cuáles fortalezas internas y externas de los jóvenes se asocian con el no consumo de tabaco? Por lo tanto, el objetivo es identificar las fortalezas internas y externas que protegen a los jóvenes del consumo de tabaco y la hipótesis es que los jóvenes que no consumen tabaco presentan mayores puntajes en algunas fortalezas internas y externas.

\section{Participantes}

La muestra fue no probabilística por conveniencia, es decir, las aplicaciones se hicieron en las escuelas que permitieron el acceso y en los grupos que estaban disponibles, los cuales fueron asignados por los directivos y estuvo constituida por 1567 estudiantes de secundaria (62\%) y bachillerato (38\%) públicos de la Ciudad de México, hombres (46.5\%) y mujeres (53.5\%) con una media de edad de 14.6 años (de=1.7). Los estudiantes de secundaria eran del turno matutino mientras que los de bachillerato eran del turno vespertino. Para ingresar a las escuelas, se agendó una cita con las orientadoras y después se realizó una entrevista 
con los directores de los planteles, los que accedieron a que se realizara la investigación.

\section{Instrumento}

Las fortalezas se evaluaron con la escala de Fortalezas Internas y Externas para Adolescentes (FIFE-A) (Betancourt, Andrade, GonzálezGonzález, \& Sánchez-Xicotencatl, 2018) que mide 7 fortalezas internas y 7 externas. Las fortalezas internas son: responsabilidad (3 reactivos, alfa $=.63$ ), importancia de la salud ( 6 reactivos, alfa $=.84$ ), evitación de conductas de riesgo ( 3 reactivos, alfa $=.86$ ), importancia de la religión ( 5 reactivos, alfa $=.93$ ), resistencia a la presión de pares ( 4 reactivos, alfa $=.77$ ), toma de decisiones ( 5 reactivos, alfa $=.87$ ) y expresión del enojo ( 4 reactivos, alfa $=.88$ ). Las fortalezas externas son: apoyo de la madre (9 reactivos, alfa $=.94$ ), supervisión de la madre ( 4 reactivos, alfa $=.85$ ), apoyo y supervisión del padre (13 reactivos, alfa $=.97)$, límites en casa $(5$ reactivos, alfa $=.75$ ), amigos sin conductas de riesgo ( 4 reactivos, alfa $=.78$ ), amigos saludables ( 4 reactivos, alfa $=.63$ ) y no acceso a drogas ( 8 reactivos, alfa $=.91)$. Los coeficientes de confiabilidad que los autores reportaron fueron aceptables.

El consumo de tabaco se evaluó con el siguiente indicador: a) nunca ha fumado tabaco; b) sí ha fumado, pero no en el último mes; c) sí ha fumado de 1 a 5 días en el último mes y d) sí ha fumado 6 o más días en el último mes. También se preguntó el número de cigarros que fumaban al día, pero no se consideró como parte del indicador, ya que la mayoría de los jóvenes (77\%) reportaron no fumar diario.

\section{Procedimiento}

Se obtuvo el permiso de las autoridades de los planteles para hacer la investigación. El instrumento se aplicó en los salones dentro del horario de clases y a los alumnos se les explicó el motivo de la investigación, contando con una participación voluntaria. El cuestionario fue anónimo. Para cumplir con los aspectos éticos se realizaron pláticas informativas con los padres de familia y estudiantes, la psicóloga escolar les explicó el motivo de la investigación y ninguno presentó alguna objeción; además se aseguró a los padres de familia y a los adolescentes que los datos eran anónimos y que no afectaría en sus actividades académicas.

\section{Análisis Estadístico}

Se utilizaron medidas de tendencia central y de desviación para describir la frecuencia de consumo de tabaco. Para describir las diferencias en las características demográficas de los jóvenes que han y no han consumido tabaco, se usó la prueba chi cuadrada en variables nominales y la $t$ de Student en variables intervalares. Para responder a la pregunta de investigación se usó el análisis de regresión logística, ya que la variable 
dependiente es dicotómica y este análisis permite identificar las variables independientes (fortalezas internas y externas) que predicen el no consumo de tabaco.

\section{Resultados}

La edad promedio de inicio del consumo de tabaco en los alumnos de secundaria tanto en hombres como en mujeres fue de 12.2 años $(\mathrm{de}=1.7)$, mientras que en bachillerato los hombres iniciaron a los de 13.6 años $(\mathrm{de}=2.1)$ y las mujeres a los $14(\mathrm{de}=1.7)$. En la Tabla 1 se muestra el patrón de consumo por sexo y grado escolar. Se observa que los alumnos de secundaria, especialmente los hombres, son los que reportan menor consumo.

Tabla 1

Consumo de tabaco por sexo y grado escolar

Tabla 1

Consumo de tabaco por sexo y grado escolar

\begin{tabular}{lcccc}
\hline & \multicolumn{2}{c}{ Secundaria (\%) } & \multicolumn{2}{c}{ Bachillerato (\%) } \\
& Hombres & Mujeres & Hombres & Mujeres \\
\cline { 2 - 5 } Nunca han fumado tabaco & 65.9 & 59.3 & 25 & 31.7 \\
Sí ha fumado, pero no en el último mes & 18.5 & 18.9 & 24.6 & 29.3 \\
$\begin{array}{l}\text { Sí ha fumado de } 1 \text { a } 5 \text { dias en el último } \\
\text { mes }\end{array}$ & 9.7 & 11.5 & 32.7 & 22.7 \\
$\begin{array}{l}\text { Sí ha fumado 6 o más dias en el último } \\
\text { mes }\end{array}$ & 5.9 & 10.3 & 17.7 & 16.3 \\
\hline
\end{tabular}

Dado que el patrón de consumo fue diferente entre los estudiantes de secundaria y preparatoria, se decidió analizar de manera separada las características sociodemográficas y las fortalezas de los estudiantes de secundaria y los de preparatoria que no han consumido tabaco y los que sí lo han hecho. En el caso de estudiantes de secundaria, se encontraron diferencias en las tres variables evaluadas; los jóvenes que no han consumido tabaco son en mayor porcentaje hombres, tienen un promedio escolar más alto y viven con ambos padres (Tabla 2). Por otro lado, en los alumnos de bachillerato sólo el promedio escolar fue diferente, y los que nunca han consumido tabaco tienen un promedio más alto (Tabla 3). 
Tabla 2

Caracteristicas sociodemográficas de los estudiantes de secundaria por consumo

Tabla 2

Características sociodemográficas de los estudiantes de secundaria por consumo

\begin{tabular}{lccc}
\hline & $\begin{array}{c}\text { No han consumido } \\
\text { tabaco } \\
(\mathrm{n}=598)\end{array}$ & $\begin{array}{c}\text { Sí han consumido } \\
\text { tabaco } \\
(\mathrm{n}=373)\end{array}$ & \\
\hline Sexo & $65.9 \%$ & $34.1 \%$ & \\
Hombres & $59.3 \%$ & $41.7 \%$ & \\
Mujeres & $8.33(.99)$ & $8.02(.91)$ & $\mathrm{x}=4.280, \mathrm{p} .039$ \\
Promedio escolar & & & \\
del último año $(d e)$ & & & \\
Tipo de familia & $74.6 \%$ & $67 \%$ & \\
Vive con ambos & & & \\
padres & $25.4 \%$ & $33 \%$ & \\
Vive con un padre & & & \\
u otros & & & \\
\hline
\end{tabular}

Tabla 3

Caracteristicas sociodemográficas de los estudiantes de bachillerato por consumo

Tabla 3

Características sociodemográficas de los estudiantes de bachillerato por consumo

\begin{tabular}{lccc}
\hline & $\begin{array}{c}\text { No han } \\
\text { consumido tabaco } \\
(\mathrm{n}=172)\end{array}$ & $\begin{array}{c}\text { Sí han consumido } \\
\text { tabaco } \\
(\mathrm{n}=424)\end{array}$ & \\
\hline Sexo & & & $\mathrm{x}^{2}=3.146, \mathrm{p} .076$ \\
Hombres & $25 \%$ & $75 \%$ & \\
Mujeres & $31.7 \%$ & $68.3 \%$ & \\
$\begin{array}{l}\text { Promedio escolar } \\
\text { del último año }(d e)\end{array}$ & $8.35(.63)$ & $8.06(.79)$ & $\mathrm{t}=4.538, \mathrm{p} .000$ \\
Tipo de familia & & & \\
Vive con ambos & $63.5 \%$ & $59.3 \%$ & \\
padres & & & \\
Vive con un padre & $36.5 \%$ & $40.7 \%$ & \\
u otros & & & \\
\hline
\end{tabular}


Con la finalidad de conocer qué fortalezas internas y externas protegen a los jóvenes del consumo de tabaco, se realizaron análisis de regresión logística binaria por grado escolar. La variable dependiente fue el consumo de tabaco y las variables independientes fueron las fortalezas internas y externas, el sexo, el tipo de familia y el promedio escolar.

El modelo de estudiantes de secundaria obtuvo un buen ajuste (Hosmer y Lemeshow=4.162, p. 842) y explicó el 43.1\% de la varianza (Nagelkerke=.431). Las fortalezas internas que resultaron protectoras del consumo de tabaco fueron la responsabilidad, resistencia a la presión de pares y expresión del enojo; mientras que las fortalezas externas fueron supervisión de la madre, amigos sin conductas de riesgo, amigos saludables y no acceso a drogas (Tabla 4).

En el caso de los estudiantes de bachillerato, el modelo presentó un buen ajuste (Hosmer y Lemeshow=8.274, p.407) y explicó el 36.2\% de la varianza (Nagelkerke=.362). Las fortalezas internas que resultaron significativas fueron la evitación de conductas de riesgo, la resistencia a la presión de pares y la importancia de la salud. Ninguna fortaleza externa resultó predictora del no consumo (Tabla 5). 
Tabla 4

Predictores del no consumo de tabaco en estudiantes de secundaria

Tabla 4

Predictores del no consumo de tabaco en estudiantes de secundaria

\begin{tabular}{|c|c|c|c|}
\hline \multirow[b]{3}{*}{ FORTALEZAS INTERNAS } & \multirow[b]{2}{*}{ OR } & \multicolumn{2}{|c|}{ Intervalo de confianza 95\% } \\
\hline & & Inferior & Superior \\
\hline & & & \\
\hline Responsabilidad & $.716^{\star}$ & .515 & .994 \\
\hline Importancia de la salud & .880 & .604 & 1.282 \\
\hline $\begin{array}{l}\text { Evitación de conductas de } \\
\text { riesgo }\end{array}$ & .839 & .703 & 1.002 \\
\hline Importancia de la religión & .930 & .772 & 1.119 \\
\hline $\begin{array}{l}\text { Resistencia a la presión de } \\
\text { pares }\end{array}$ & $.372^{\star \star \star}$ & .221 & 627 \\
\hline Toma de decisiones & .857 & 659 & 1.115 \\
\hline Expresión del enojo & $.807^{\star}$ & .662 & .984 \\
\hline FORTALEZAS EXTERNAS & & & \\
\hline Apoyo de la madre & 1.102 & .839 & 1.447 \\
\hline Supervisión de la madre & $.714^{\star}$ & .512 & .995 \\
\hline Apoyo y supervisión del padre & .949 & .772 & 1.167 \\
\hline Límites en casa & 1.003 & .769 & 1.307 \\
\hline $\begin{array}{l}\text { Amigos sin conductas de } \\
\text { riesgo }\end{array}$ & $.574^{\star \star \star}$ & .454 & .726 \\
\hline Amigos saludables & $1.456^{\star}$ & 1.052 & 2.016 \\
\hline No acceso a drogas & $.573^{\star \star \star}$ & .447 & .734 \\
\hline $\mathrm{SexO}^{+}$ & .754 & .480 & 1.186 \\
\hline Tipo de familia ${ }^{++}$ & .990 & 612 & 1.603 \\
\hline Promedio escolar & .817 & .662 & 1.008 \\
\hline
\end{tabular}

Nota: Categoría de referencia: +hombre, ++ vive con ambos padres. ${ }^{*} \mathrm{p}>.05^{* *} \mathrm{p}>.01{ }^{* *} \mathrm{p}>.001$ 


\section{Tabla 5}

Predictores del no consumo de tabaco en estudiantes de bachillerato

Tabla 5

Predictores del no consumo de tabaco en estudiantes de bachillerato

\begin{tabular}{|c|c|c|c|}
\hline \multirow[b]{3}{*}{ FORTALEZAS INTERNAS } & \multirow[b]{2}{*}{ OR } & \multicolumn{2}{|c|}{ Intervalo de confianza 95\% } \\
\hline & & Inferior & Superior \\
\hline & & & \\
\hline Responsabilidad & .931 & .611 & 1.419 \\
\hline Importancia de la salud & $1.901^{\star \star}$ & 1.175 & 3.075 \\
\hline $\begin{array}{l}\text { Evitación de conductas de } \\
\text { riesgo }\end{array}$ & $.605^{\star \star}$ & .438 & .836 \\
\hline Importancia de la religión & .923 & .730 & 1.167 \\
\hline $\begin{array}{l}\text { Resistencia a la presión de } \\
\text { pares }\end{array}$ & $.168^{\star \star \star}$ & .065 & .433 \\
\hline Toma de decisiones & .837 & .584 & 1.202 \\
\hline Expresión del enojo & .955 & .727 & 1.254 \\
\hline FORTALEZAS EXTERNAS & & & \\
\hline Apoyo de la madre & .780 & .530 & 1.148 \\
\hline Supervisión de la madre & 1.102 & .694 & 1.751 \\
\hline Apoyo y supervisión del padre & 1.211 & .933 & 1.573 \\
\hline Límites en casa & .732 & .518 & 1.034 \\
\hline $\begin{array}{l}\text { Amigos sin conductas de } \\
\text { riesgo }\end{array}$ & .757 & .543 & 1.056 \\
\hline Amigos saludables & .999 & .657 & 1.520 \\
\hline No acceso a drogas & .779 & .588 & 1.032 \\
\hline $\mathrm{Sexo}^{++}$ & .609 & .322 & 1.155 \\
\hline Tipo de familia $^{+}$ & .828 & .446 & 1.536 \\
\hline Promedio escolar & .716 & .48 .2 & 1.062 \\
\hline
\end{tabular}

Nota: Categoría de referencia: + hombre ++ vive con ambos padres. ${ }^{*} \mathrm{p}>.05^{* *} \mathrm{p}>.01{ }^{* * *} \mathrm{p}>.001$

\section{Discusión}

En México, si bien el consumo de tabaco en los últimos años ha disminuido ligeramente entre los jóvenes, el porcentaje de consumidores aún es elevado. Además, si se considera la edad en que inician el consumo y los daños a los que se exponen, es necesario continuar con estudios que expliquen las variables asociadas, con el fin de diseñar programas de prevención eficaces. En este estudio se corroboró lo reportado en la Encuesta Nacional de Consumo de Drogas en Estudiantes (Instituto 
Nacional de Psiquiatría, Comisión Nacional contra las Adicciones, Secretaría de Salud, 2015), en lo que se refiere a que conforme aumenta la edad el consumo de tabaco se incrementa, pero contradice el dato referente a que los hombres presentan un consumo mayor, ya que las estudiantes de secundaria informaron que consumen más que los hombres.

En cuanto a las fortalezas internas, la habilidad de resistencia a la presión de pares fue una variable que favorece que los jóvenes tanto de secundaria como de preparatoria no consuman tabaco. Es importante mencionar que esta fortaleza ha sido reportada en diferentes estudios como un factor protector del consumo (Andrade et al., 2009; Gibbon et al., 2014; Hopfer et al., 2013), por lo cual es necesaria su inclusión en programas de prevención tanto universales como selectivos e indicados. Otras fortalezas internas protectoras del consumo en los estudiantes de secundaria fueron la percepción de responsabilidad, que se refiere básicamente a si el joven cumple sus compromisos y es puntual, y la expresión del enojo, que hace referencia a si el joven no se percibe como enojón o con carácter irritable. La expresión del enojo ya había sido reportada en otros estudios (Alfaro et al., 2010; Bernstein et al., 2014), no obstante, es importante no sólo evaluar si el joven se percibe enojón o irritable, sino más bien hay que enfocarse en cómo maneja su enojo.

En el caso de los estudiantes de preparatoria, las fortalezas internas que obtuvieron asociaciones significativas son la importancia que los jóvenes dan a su salud y a evitar conductas de riesgo, como el consumo de sustancias. Estos resultados confirman lo que Andrade y SánchezXicotencatl (2018) encontraron en estudiantes mexicanos. Además, confirman de alguna manera los resultados de otros autores que señalan la importancia que tiene la percepción de los daños que ocasiona el tabaco (Cable et al., 2017; Kasim et al., 2016), pues si bien es cierto que la información por sí sola no produce cambios en la conducta, sí es importante dar esta información a los jóvenes. Sin embargo, también es relevante enfatizar los riesgos que se tienen y el rol que ellos juegan en cuanto a decidir cómo pueden cuidar su salud (Pilatti et al., 2017).

Respecto a las fortalezas externas, es importante señalar que en los estudiantes de preparatoria de esta muestra no se encontraron asociaciones significativas, a pesar de que en diversos estudios se ha identificado la influencia de los amigos como uno de los principales factores asociados al consumo de drogas, incluyendo al tabaco (Chun \& Chung, 2013; Jaber et al., 2016). Estos resultados contradicen lo reportado por Andrade y Sánchez-Xicotencatl (2018) quienes encontraron que la fortaleza de tener amigos sin conductas de riesgo es un predictor del no consumo de tabaco en estudiantes de preparatoria. También contradicen lo reportado por Kobus (2003) en cuanto a que los jóvenes que tienen amigos que no consumen tabaco son menos propensos a consumirlo. Es necesario continuar con investigaciones que permitan confirmar o refutar estos resultados.

En el caso de los estudiantes de secundaria, esta investigación corrobora lo reportado por Atkins, Oman, Vesely, Aspy y McLeroy (2002), quienes 
identificaron que tener amigos que tienen conductas saludables es una fortaleza externa que incrementa la probabilidad de que los jóvenes no consuman tabaco. También confirma lo reportado por otros autores respecto a la influencia de los amigos (Chun \& Chung, 2013; Jaber et al., 2016).

Otra fortaleza externa protectora en los estudiantes de secundaria fue tener amigos saludables, es decir, tener amigos que hacen ejercicio, duermen suficiente, etcétera. Esta fortaleza no se ha reportado en otros estudios, ya que la mayoría se enfoca en los amigos que consumen. El no tener acceso a drogas también se asoció significativamente en los estudiantes de secundaria, resultado que coincide con otros estudios (Andrade \& Sánchez-Xicotencatl, 2018; Lido\#n-Moyano et al., 2017; Pabayo et al., 2012), y que muestra la importancia de implementar políticas públicas dirigidas a establecer normas antitabaco, sobre todo en menores de edad.

La fortaleza externa referente a la supervisión familiar ha sido ampliamente documentada en la literatura (Hiemstra et al., 2017; Kim \& Chun, 2016). En este estudio sólo en los estudiantes de secundaria se asoció significativamente con el no consumo de tabaco, lo cual se puede explicar porque los adolescentes tempranos tienen una supervisión más estrecha de los padres que los adolescentes tardíos.

En esta investigación no se corroboraron los resultados de otros autores que reportan como protectores del consumo de tabaco a la fortaleza interna de religión (Fletcher \& Kumar, 2014; Nonnemaker et al., 2006; Sinha et al., 2007) ni a la toma de decisiones. Tampoco fueron significativas las fortalezas externas de apoyo del padre y de la madre y límites en casa, las cuales han sido reportadas en otros estudios (Hiemstra et al., 2017; Piko \& Balázs, 2012). Estas aparentes contradicciones pueden deberse a las diferentes formas en que se evalúan; por ejemplo, en este estudio la fortaleza de límites en casa hace referencia a si existen horarios en casa para comer, dormir, etcétera, mientras que en otros estudios se refieren a reglas como prohibir el consumo de tabaco dentro de casa.

Es preciso señalar que las fortalezas tanto internas como externas que mostraron mayor probabilidad de no consumo de tabaco en los estudiantes de secundaria no fueron las mismas que en los estudiantes de preparatoria, lo cual señala la importancia de hacer estudios por grupos de edad.

\section{Limitaciones}

Una de las principales limitaciones del estudio es el tipo y tamaño de la muestra, ya que no se tuvo una muestra aleatoria y representativa de los jóvenes, por lo cual los resultados no pueden generalizarse a los jóvenes mexicanos. Se requiere replicar el estudio con otras muestras en donde se incluyan no sólo estudiantes. Otra posible limitación es que no se abarcan todas las fortalezas que pudieran explicar por qué los jóvenes no consumen tabaco. Quizá sería conveniente incluir algunos aspectos relacionados con 
la forma en que los adolescentes afrontan el estrés debido a los cambios que se presentan en esta etapa.

\section{Conclusiones}

En México es poca la investigación que se enfoca en los factores que protegen a los jóvenes del consumo de tabaco, por lo que este estudio, además de aportar evidencia empírica del enfoque de fortalezas (Benson et al., 2006), contribuye a identificar los aspectos positivos del joven y su entorno para incidir en el diseño de programas de prevención. No obstante, se requiere continuar con investigaciones que incluyan a jóvenes que no estudian o que están en situación de riesgo para tener una mejor explicación del fenómeno del consumo de tabaco y tener más elementos para el diseño de programas de promoción de la salud.

\section{Agradecimientos}

Este estudio fue financiado por el proyecto CONACYT 247574 "Programa de promoción de la salud en estudiantes de secundaria". Los autores no tienen conflictos de interés.

\section{Referencias}

Alfaro, L. B., Sánchez, M. E., Andrade, P., Pérez, C., \& Montes, A. (2010). Habilidades para la vida, consumo de tabaco y alcohol en adolescentes. Revista Española de Drogodependencias, 35(1), 67-77.

Andrade, P., \& Sánchez-Xicotencatl, C.O. (2018). Fortalezas protectoras del consumo de alcohol y tabaco. En P. Andrade (coord). Fortalezas de los Adolescentes (pp. 61-80). CDMX, México: Universidad Nacional Autónoma de México.

Andrade, P., Pérez, C., Alfaro, L., Sánchez, M., \& Montes, A. (2009). Resistencia a la presión de pares y pareja y consumo de tabaco y alcohol en adolescentes. Adicciones, 21(3), 243-250. https://doi.org/10.20882/adicciones.235

Atkins, L. A., Oman, R. F., Vesely, S. K., Aspy, C. B., \& McLeroy, K. (2002). Adolescent tobacco use: The protective effects of developmental assets. American Journal of Health Promotion, 16(4), 198-205. https://doi.org/1 0.4278/0890-1171-16.4.198

Benson, P. (2007). Developmental assets: An overview of theory, research, and practice. En R. Lerner \& R. Silbereisen, (Eds.), Approaches to positive youth development (pp. 31-58). Thousand Oaks, CA, Estados Unidos: Sage Publications.

Benson, P. L., Scales, P. C., Hamilton, S. H., \& Sesma, A. Jr. (2006). Positive youth development: Theory, research and applications. En W. Damon \& R. M. Lerner (Eds.), Handbook of child psychology (Vol. 1, 6th ed., pp. 894-941). Nueva York, NY, Estados Unidos: Wiley.

Bernstein, M. H., Colby, S. M., Bidwell, L. C., Kahler, C. W., \& Leventhal, A. M. (2014). Hostility and cigarette use: A comparison between smokers 
and nonsmokers in a matched sample of adolescents. Nicotine \& Tobacco Research, 16(8), 1085-1093. https://doi.org/10.1093/ntr/ntu033

Betancourt, D., Andrade, P., González-González, A., \& Sánchez-Xicotencatl, C. O. (2018). Escala de fortalezas para adolescentes (FIE-A). En P. Andrade (coord). Fortalezas de los Adolescentes (pp. 35-60). CDMX, México: Universidad Nacional Autónoma de México.

Brikmanis, K., Petersen, A., \& Doran, N. (2017). Do personality traits related to affect regulation predict other tobacco product use among young adult non-daily smokers? Addictive Behaviors, 75, 79-84. https://doi.org/10.1 016/j.addbeh.2017.07.008

Cable, N., Roman, M. F., \& Kelly, Y. (2017). What could keep young people away from alcohol and cigarettes? Findings from the UK Household Longitudinal Study. BMC Public Health, 17, 371. https://doi.org/10.11 86/s12889-017-4284-x

Chun, J., \& Chung, I. (2013). Gender differences in factors influencing smoking, drinking, and their co-occurrence among adolescents in South Korea. Nicotine \& Tobacco Research, 15(2), 542-551. https://doi.org/10. $1093 / \mathrm{ntr} / \mathrm{nts} 181$

DiNapoli, P. (2009). Early initiation of tobacco use in adolescent girls: Key sociostructural influences. Applied Nursing Research, 22(2), 126-132. htt ps://doi.org/10.1016/j.apnr.2007.07.001

Fletcher, J., \& Kumar, S. (2014). Religion and risky health behaviors among U.S. adolescents and adults. Journal of Economic Behavior \& Organization, 104, 123-140. https://doi.org/10.1016/j.jebo.2014.03.018

Gibbon, L., Griffin, K. W., Tanno, S., Tanigawa, T., \& Botvin, G. J. (2014). Perceived friend and peer smoking and direct and indirect refusal skills as predictors of cigarette smoking in U.S. and Japanese middle school students. Journal of Ethnicity in Substance Abuse, 13(3), 209-226. https:/ /doi.org/10.1080/15332640.2013.847394

Heck, K. E., \& Subramaniam, A. (2009). Youth development frameworks. Davis, CA, Estados Unidos: Centre for Youth Development, UCLA Press. Recuperado de http://4h.ucanr.edu/files/1224.pdf

Hiemstra, M., de Leeuw, R. N., Engels, R. C., \& Otten, R. (2017). What parents can do to keep their children from smoking: A systematic review on smoking-specific parenting strategies and smoking on set. Addictive Behaviors, 70, 107-128. https://doi.org/10.1016/j.addbeh.2017.02.003

Hopfer, S., Hecht, M. L., Lanza, S. T., Xianming, T., \& Xu, S. (2013). Preadolescent drug use resistance skill profiles, substance use, and substance use prevention. The Journal of Primary Prevention, 34(6), $395-$ 404. https://doi.org/10.1007/s10935-013-0325-0

Instituto Nacional de Psiquiatría Ramón de la Fuente Muñiz; Comisión Nacional Contra las Adicciones; Secretaría de Salud. (2015). Encuesta Nacional de Consumo de Drogas en Estudiantes 2014: Reporte de Drogas. CDMX, México: INPRFM.

Jaber, R., Mzayek, F., Madhivanan, P., Khader, Y., \& Maziak, W. (2016). Predictors of cigarette smoking progression among a school-based sample of adolescents in Irbid, Jordan: A longitudinal study (2008-2011). Nicotine \& Tobacco Research, 18(4), 403-409. https://doi.org/10.1093/n $\operatorname{tr} / \mathrm{ntv} 100$ 
Kasim, K., Al-Zalabani, A., Abd El-Moneim, E., \& Amer, S. (2016). Beliefs and attitudes of male and female adolescents and the risk of smoking behavior. Journal of Postgraduate Medicine, 62(2), 80-85. https://doi.org/10.4103/ 0022-3859.180546

Kim, H. H., \& Chun, J. (2016). Examining the effects of parental influence on adolescent smoking behaviors: A multilevel analysis of the Global SchoolBased Student Health Survey (2003-2011). Nicotine \& Tobacco Research, 18(5), 934-942. https://doi.org/10.1093/ntr/ntv172

Kleinjan, M., van den Eijnden, R. J., \& Engels, R. C. (2009). Adolescents' rationalizations to continue smoking: The role of disengagement beliefs and nicotine dependence in smoking cessation. Addictive Behaviors, 34(5), 440-445. https://doi.org/10.1016/j.addbeh.2008.12.010

Kobus, K. (2003). Peers and adolescent smoking. Addiction, 98(1), 37-51. http s://doi.org/10.1046/j.1360-0443.98.s1.4.x

Lido\#n-Moyano, C., Fu, M., Ballbe\#, M., Marti\#n-Sa\#nchez, J. C., MatillaSantandera, N., Marti\#nez, C., ... \& Marti\#nez-Sa\#nchez, J. M. (2017). Impact of the Spanish smoking laws on tobacco consumption and secondhand smoke exposure: A longitudinal population study. Addictive Behaviors, 75, 30-35. https://doi.org/10.1016/j.addbeh.2017.06.016

Mays, D., Gilman, S. E., Rende, R., Luta, G., Tercyak, K. P., \& Niaura, R. S. (2014). Parental smoking exposure and adolescent smoking trajectories. Pediatrics, 133(6), 983-991. https://doi.org/10.1542/peds.2013-3003

Ng, M., Freeman, M. K., Fleming, T. D., Robinson, M., Dwyer-Lindgren, L., Thomson, B., ... Gakidou, E. (2014). Smoking prevalence and cigarette consumption in 187 countries, 1980-2012.JAMA, 311(2), 183-192. htt ps://doi.org/10.1001/jama.2013.284692

Nonnemaker, J. M., McNeely, C. A., \& Blum, R. W. (2006). Public and private domains of religiosity and adolescent smoking transitions. Social Science \& Medicine, 62(12), 3084-3095. https://doi.org/10.1016/j.socscimed.20 05.11 .052

Nuño-Gutiérrez, B., Álvarez-Nemegyei, J., Velázquez-Castañeda, A., \& TapiaCuriel, A. (2008). Comparación del ambiente familiar y el tipo de consumo de tabaco en adolescentes mexicanos de nivel medio superior. Salud Mental, 31(5), 361-369.

Organización Panamericana de la Salud (OPS). (2018). Informe sobre el control del tabaco en la Región de las Américas. Washington, DC, Estados Unidos: OPS.

Pabayo, R., O’Loughlin, J., Barnett, T. A., Cohen, J. E., \& Gauvin, L. (2012). Does intolerance of smoking at school, or in restaurants or corner stores decrease cigarette use initiation in adolescents? Nicotine \& Tobacco Research, 14(10), 1154-1160. https://doi.org/10.1093/ntr/ntr326

Piko, B. F., \& Balázs, M. A. (2012). Authoritative parenting style and adolescent smoking and drinking. Addictive Behaviors, 37(3), 353-356. https://doi.o $\mathrm{rg} / 10.1016 /$ j.addbeh.2011.11.022

Pilatti, A., Read, J. P., \& Pautassi, R. M. (2017). ELSA 2016 cohort: Alcohol, tobacco, and marijuana use and their association with age of drug use onset, risk perception, and social norms in Argentinean college freshmen. Frontiers in Psychology, 8, 1452. https://doi.org/10.3389/fpsyg.2017.014 52 
Sinha, J. W., Cnaan, R. A., \& Gelles, R. J. (2007). Adolescent risk behaviors and religion: Findings from a national study.Journal of Adolescence, 30(2), 231-249. https://doi.org/10.1016/j.adolescence.2006.02.005

Veeranki, S. P., Alzyoud, S., Dierking, L., Kheriallah, K., Mzayek, F., Pbert, L., \& Ward, K. D. (2015). Associations of adolescents' cigarette, waterpipe, and dual tobacco use with parental tobacco use. Nicotine \& Tobacco Research, 18(5), 879-884. https://doi.org/10.1093/ntr/ntv224 